\title{
LA EFECTIVIDAD DEL FEEDBACK CORRECTIVO ESCRITO INDIRECTO METALINGÜÍSTICO EN EL ESPAÑOL COMO LENGUA EXTRANJERA
}

\section{THE EFFECTIVENESS OF METALINGUISTICS INDIRECT WRITTEN FEEDBACK CORRECTIVE IN SPANISH AS A FOREIGN LANGUAGE}

\section{Anita Alejandra Ferreira Cabrera*}

\section{RESUMEN}

La propuesta de estrategias de Feedback Correctivo Escrito (FCE) para mejorar la precisión en la escritura requiere de investigaciones que examinen los efectos de recibir versus no recibir FCE en el corto y largo plazo. En este trabajo, se presenta un estudio experimental longitudinal con pre-test/post-test (inmediato y diferido) y grupo control para evaluar la efectividad del FCE indirecto metalingüístico y directo metalingüístico en la disminución de errores en aprendientes de nivel A2 y B1 en ELE. Los errores tratados corresponden a la ortografía acentual, las preposiciones y la concordancia gramatical. Los resultados muestran una mayor efectividad en el FCE indirecto metalingüístico tanto en los niveles de competencia A2 como B1 en el corto y largo plazo.

Palabras claves: Feedback Correctivo Escrito; Feedback Correctivo Escrito Indirecto Metalingüístico; Español como Lengua Extranjera.

\section{ABSTRACT}

The proposal of Written Corrective Feedback (WCF) strategies that aim at improving writing accuracy requires research that examines the short and long term effects of receiving versus not receiving WCF. This article presents a longitudinal experimental study with pretest/post-test (immediate and delayed) and control group with the aim of evaluating the effectiveness of direct and indirect metalinguistic WCF in diminishing errors in A2 and B1 learners of Spanish as FL. Treated errors correspond to accentual orthography, prepositions and grammatical concordance. The findings indicate that the WCF resulted in being more effective rather than not working without such linguistic support.

Keywords: Written Corrective Feedback, Indirect Metalinguistics Written Corrective Feedback; Spanish as a Foreign Language.

\footnotetext{
* Universidad de Concepción, Chile. aferreir@udec.cl 


\section{INTRODUCCIÓN}

La enseñanza de la destreza escrita en Español como Lengua Extranjera (ELE), en un contexto Centrado en la Forma (Focus on Form), requiere de estrategias de enseñanza y de apoyos metodológicos innovadores que faciliten el tratamiento de los errores en la destreza escrita en ELE (FERREIRA, MOORE \& MELLISH, 2007). El Enfoque centrado en la Forma (LONG \& ROBINSON, 1998; ELLIS, 2016) aborda la pregunta sobre cómo se deben distribuir los recursos de atención focalizados en las formas lingüísticas que deberían tratarse y la atención en el significado del texto o discurso. El propósito es facilitar la atención de los errores lingüísticos que se perciben en la producción del texto. En esta perspectiva, el Feedback Correctivo Escrito (FCE) se constituye en una modalidad de enseñanza de la lengua ampliamente utilizada por los profesores para hacer notar a los alumnos las formas incorrectas y promover la producción posterior de las formas correctas. La precisión en la escritura es de vital importancia en las audiencias académicas y profesionales, en las cuales los errores pueden estigmatizar a quienes escriben (FERRIS, 2004). El tema de la efectividad del feedback en la habilidad escrita de los aprendientes de segundas lenguas ha sido objeto de estudio y controversia en las últimas décadas (VAN BEUNINGEN, 2010; FERRIS, 2010; SHEEN, 2011; BITCHENER \& FERRIS, 2012). El punto central de la investigación ha estado en poder determinar si las estrategias de FCE ayudan a mejorar la precisión escrita de los estudiantes.

En efecto, el feedback correctivo también conocido como "corrección gramatical" o "corrección del error" ha sido un tema crucial en el ámbito de la adquisición de segundas lenguas, tanto para estimular como para consolidar el aprendizaje en la habilidad escrita (FERRIS, 2012; BITCHENER, 2012). Una de las primeras definiciones de feedback correctivo es la que aporta Chaudron (1977), quien lo definió como: "cualquier reacción del profesor que claramente transforma, desaprueba o pide mejorar el enunciado del estudiante". En investigaciones más recientes, Sheen (2011) utiliza el término para referirse a la corrección de errores léxico-gramaticales, definiéndolo como "aquel que sigue a una respuesta incorrecta" (gramaticalmente incorrecta)".

Las investigaciones sobre el FCE metalingüístico han examinado los efectos de recibir versus no recibir estrategias en el corto y largo plazo (BITCHENER \& KNOCH, 2010; VAN BEUNINGEN, 2010; ELLIS, 2009; SHEEN, 2011). Este tipo de estrategias ayuda a desarrollar la conciencia metalingüística a través de una atención selectiva de errores enfocados en el proceso de escritura con el objeto de mejorar la precisión lingüística. Se trata de elevar el nivel de conciencia lingüística de los aprendientes, llamándoles la atención sobre sus errores sistemáticos, a través 
de información metalingüística clave para que noten o perciban sus problemáticas y mejoren la comprensión de ellas, optimizando así su habilidad escrita. Sin embargo, los estudios no han podido dar una respuesta definitiva sobre la efectividad de dichas estrategias correctivas. Al respecto, se ha examinado si el FCE facilita la adquisición de rasgos lingüísticos particulares a largo plazo, cómo estos se adquieren y cuántos deben ser estudiados; también se ha abordado si el feedback debe ser directo o indirecto, y cuánta explicación metalingüística es necesaria (BITCHENER, 2009, ELLIS, 2009; VAN BEUNINGEN, 2010; SHEEN, 2011), todo ello apoyado por el paradigma cognitivo de la ASL. Ellis (2009) señala que el FC metalingüístico lleva al estudiante a formular reglas sobre ciertos rasgos gramaticales, pero les toma tiempo el ser capaces de usar estas reglas de manera efectiva.

Otra distinción relevante en materia de investigación sobre el FCE se refiere al tratamiento de los errores a través de un feedback focalizado versus uno no focalizado. Este último, corresponde a la práctica habitual de los profesores, quienes corrigen todos los errores en los trabajos escritos de los estudiantes (ELLIS, SHEEN, MURAKAMI \& TAKASHIMA, 2008), en contraste con el FCE focalizado que selecciona errores específicos para ser corregidos y descarta otros. Un FCE altamente focalizado se va a concentrar en una, a lo más dos categorías. Según Ellis, Sheen, Murakami y Takashima (2008), existen razones teóricas sólidas que avalan el argumento de que el FCE focalizado puede ser más acertado que el FCE no focalizado. Los estudiantes son más sensibles en poner atención a correcciones dirigidas a uno o a un número limitado de tipos de errores para desarrollar mayor comprensión de la naturaleza del error y la corrección necesaria. Si la atención (noticing) y el entendimiento (SCHMIDT, 2010) son necesarios para la adquisición, entonces el FCE focalizado está claramente en mejores condiciones para producir mejores resultados. La evidencia obtenida por los investigadores (SHEEN, 2007; BITCHENER, 2009; BITCHENER \& KNOCH, 2009a) dan cuenta de la eficacia del FCE focalizado.

Nuestro enfoque de investigación se fundamenta en la necesidad de tratar el error a lo largo del proceso de adquisición y aprendizaje de una lengua extranjera (LE), porque este forma parte del mismo y, por lo tanto, es una evidencia de este proceso. El objetivo principal en el presente artículo es evaluar los efectos de las estrategias de FCE indirecto y directo metalingüístico en el proceso de escritura de aprendientes de niveles A2 y B1 en el Español como Lengua Extranjera. El FCE directo metalingüístico se entiende como la provisión de la información lingüística necesaria para corregir el error con la provisión de la forma correcta. En cambio, el FCE indirecto metalingüístico indica la presencia de un error y lo elicita 
a través de información metalingüística, pero sin dar la respuesta correcta. En esta investigación, ambos tipos de FCE se focalizan en errores de ortografía acentual, preposiciones y concordancia gramatical. En este contexto empírico, se presenta un estudio experimental longitudinal en el cual participaron 77 estudiantes de Español como Lengua Extranjera de niveles A2 y B1. El artículo se organiza en las siguientes secciones: En la sección 1, nos referimos a los principales fundamentos teóricos sobre el Feedback Correctivo Escrito. En la sección 2, abordamos el estudio sobre el FCE indirecto y directo metalingüístico. En la sección 3, presentamos el análisis y discusión de los resultados de la investigación. Finalmente, en la sección 4 presentamos algunos comentarios finales y proyecciones sobre los avances y logros obtenidos en esta investigación.

\section{FUNDAMENTOS TEÓRICOS}

\subsection{Perspectivas en torno al Feedback Correctivo Escrito}

Las investigaciones sobre FCE se han desarrollado principalmente en torno a dos perspectivas, cada una de ellas con su propia orientación teórica y metodológica: el campo de la escritura en la L2, y el dominio de Adquisición de Segundas Lenguas (ASL). Aunque ambas perspectivas examinan aspectos similares del FC escrito, estas apuntan a responder interrogantes distintas. El interés de la investigación en el campo de la escritura en la L2 ha tratado de responder la interrogante de si el FCE ayuda a los estudiantes a mejorar la efectividad general de los textos producidos, y si es así, cómo es que ocurre. En cambio, los investigadores en el ámbito de la ASL han estado averiguando sobre la efectividad inmediata y a largo plazo del FCE en condiciones experimentales. El propósito ha sido indagar si este facilita la adquisición de rasgos lingüísticos particulares en un marco de la enseñanza con atención a la forma. De esta manera, en el dominio de la ASL, la investigación sobre la efectividad intenta determinar el potencial que tiene el FC en general y los diferentes tipos de FC para favorecer el desarrollo de la interlengua (FERRIS, 2010; VAN BEUNINGEN, 2010; BITCHENER, 2012; LOEWEN, 2012).

Por otra parte, desde la enseñanza-aprendizaje de una L2, las preguntas se han centrado en delimitar si el FCE ayuda a los estudiantes a mejorar la precisión general de sus textos y a desarrollarse como escritores exitosos. Para poder fundamentar que la corrección de errores apoya el aprendizaje, se debe examinar si el mejoramiento dado en las revisiones de los textos se transfiere en mediciones realizadas en posttest inmediatos y diferidos. En la actualidad, existe necesidad de realizar estudios que 
permitan examinar los efectos de recibir versus no recibir FCE en el corto y largo plazo, que arrojen evidencias más concluyentes a favor de la efectividad del FCE en la precisión de la lengua escrita.

\subsection{La efectividad del Feedback Correctivo Escrito (FCE) directo e indirecto}

En relación con la efectividad del FCE, los estudios han tratado de resolver cómo y por qué diferentes tipos de FCE, algunos más explícitos que otros, podrían llevar a los estudiantes a progresar en el aprendizaje y en la precisión lingüística.

La investigación ha abordado el efecto de diferentes tipos de FCE en la destreza escrita y la medida en que dichos efectos son mantenidos a través del tiempo. Los autores que apoyan el FCE indirecto han sugerido que es un enfoque más efectivo porque requiere que el estudiante se comprometa cognitivamente en el aprendizaje guiado, la resolución de problemas y la autocorrección de sus errores, por tanto, promovería el tipo de atención y reflexión que tiende más a la adquisición en el largo plazo. Aquellos que han demostrado estar a favor de la corrección directa sugieren que esta (1) reduce el tipo de confusión que los aprendientes podrían experimentar si fallan en entender las correcciones indirectas (brindadas, por ejemplo, mediante códigos de error o enumeración de errores al margen), (2) brinda la información necesaria para la resolución de errores más complejos, (3) ofrece una retroalimentación más explícita para la comprobación de hipótesis y (4) es más inmediato (CHANDLER, 2003; ELLIS, 2008; BITCHENER \& KNOCH, 2010).

Aunque estos términos no siempre se han utilizado constantemente en la literatura, el FCE directo puede ser definido como la provisión de la forma correcta o de la información lingüística necesaria para corregir el error cometido (FERRIS, 2004; ELLIS, 2008; SHEEN, 2011). Este puede incluir el tachar una palabra, frase o morfema que no sea necesario, la inserción de una palabra, frase o morfema o la provisión de la forma correcta (BITCHENER, 2009). Otras formas de FCE directo pueden ser una explicación metalingüística escrita o una explicación metalingüística oral. Por otra parte, el FCE indirecto indica de alguna manera, que se ha cometido un error. Esto puede ser subrayando el error, entregando información metalingüística, registrando al margen la cantidad de errores en una línea determinada, o utilizando un código para mostrar que el error ha ocurrido y qué tipo de error es (HYLAND \& HYLAND, 2006; ELLIS, 2009; SHEEN, 2011); en este caso, son los estudiantes quienes deben resolver y corregir el problema en el cual se ha focalizado su atención. 
Ferris y Roberts (2001) sugieren que el FCE directo es más apropiado que el FCE indirecto para los estudiantes que tienen un nivel de competencia lingüística inicial en la L2, ya que su nivel de conocimiento lingüístico no es suficiente para auto corregirse. Sin embargo, este tipo de feedback tiene la desventaja de demandar un mínimo de procesamiento cognitivo debido a que el estudiante no tiene que detenerse a meditar donde está el error o a qué se debe éste, sino que simplemente debe notarlo (SCHMIDT, 2010). De esta forma, si se considera que el aprendizaje demanda un alto nivel de procesamiento cognitivo, aún cuando el FCE directo pueda ayudar al estudiante a notar el error y producir la forma correcta al momento de realizar la modificación de su texto el solo hecho de notarlo puede ser insuficiente para su aprendizaje en el largo plazo al escribir un nuevo texto en el futuro.

Por otra parte, las ventajas del FCE indirecto, según los investigadores, radican en que éste obliga al estudiante a involucrarse en un aprendizaje guiado con resolución de problemas, los estimula a reflexionar sobre las formas lingüísticas (ELLIS, 2009) y los ayuda a desarrollar habilidades para llegar a ser "autoeditores independientes" (HYLAND \& HYLAND, 2006; SHEEN, 2011; VAN BEUNINGEN, 2010). Por esta razón, se piensa que este tipo de estrategia de FCE puede conducir al aprendizaje en el largo plazo (FERRIS \& ROBERTS, 2001). BITCHENER (2009) menciona que existen pocos estudios en FCE que comparen resultados entre FCE directo y FCE indirecto, y que en estudios preliminares hubo inclinación por el segundo debido a la capacidad que genera en los estudiantes el reflexionar acerca de los errores, lo que puede promover la adquisición a largo plazo. Algunos teóricos (FERRIS, 2010; SHEEN, 2011), sin embargo, que no desmerecen el valor del FCE indirecto estiman que el directo es preferido por profesores y alumnos, además sugieren que el FCE directo reduce el tipo de confusión que resulta cuando los estudiantes no logran comprender o recordar los códigos utilizados por el profesor. Bitchener (2009) sostiene que analizando ventajas y desventajas, no es posible determinar cuál de los dos tipos de feedback es mejor. Probablemente, en lo referido hasta aquí, lo más indicado sea averiguar los contextos y las características individuales observables en los alumnos para delimitar con mayor claridad el efecto de estas estrategias en el proceso de la escritura y la precisión lingüística en los textos.

Las investigaciones subsecuentes realizadas por Bitchener y Knoch (2009b) no arrojaron diferencias en la efectividad del FCD cuando es acompañado o no por clave metalingüística. Si bien dichas estrategias han resultado ser las preferidas por los aprendientes (BITCHENER \& KNOCH, 2010). En definitiva, las investigaciones más recientes en la adquisición de segundas lenguas (ASL) en materia de efectividad, 
en lo referido al contraste entre los tipos de FCE directos versus indirectos, no permiten establecer resultados concluyentes respecto de sus efectos inmediatos o si estos se extienden a tareas futuras. En consecuencia, existe necesidad de explorar nuevas perspectivas de investigación en torno a estas problemáticas que permitan tanto complementar los hallazgos hasta ahora encontrados como al mismo tiempo enriquecerlos con nuevas evidencias y propuestas metodológicas innovadoras en el ámbito de la adquisición y enseñanza de la destreza escrita en ELE.

\section{EL ESTUDIO}

Con el fin de evaluar en la enseñanza del Español como Lengua Extranjera los efectos de las estrategias de FCE indirecto metalingüístico y directo metalingüístico se llevó a cabo un estudio empírico basado en un diseño experimental longitudinal con pre-test/post-test (inmediato y diferido) y grupo control.

\subsection{Pregunta de investigación}

La pregunta de investigación que orienta este estudio es: ¿ Qué efectos tienen a corto y largo plazo las estrategias de FCE indirecto metalingüístico y directo metalingüístico en el mejoramiento de la precisión lingüística en la destreza escrita en el Español como Lengua Extranjera?

\subsection{Hipótesis: en relación con dicha pregunta se precisaron las siguientes hipótesis de trabajo:}

1) Los errores de los aprendientes de ELE que sean tratados con estrategias de FCE indirecto metalingüístico y directo metalingüístico en un corto y largo plazo evidenciarán una mayor disminución en comparación con los errores de los aprendientes del grupo control, que sólo recibirán comentarios generales (culturales y léxicos) en relación con sus textos.

2) Los errores de los estudiantes tratados con FCE indirecto metalingüístico evidenciarán una mayor disminución en el proceso de corrección que los tratados con FCE directo metalingüístico tanto en el corto como en el largo plazo.

\subsection{La muestra}

La muestra se compone por un total de 77 aprendientes de ELE de una universidad chilena: 38 con un nivel de competencia A2 y 39 con un nivel B1. Para 
corroborar el nivel de competencia declarado por los estudiantes, se aplicó la Prueba de Nivel de Competencia en ELE (FERREIRA,VINE Y ELEJALDE, 2013) del Programa de Español como Lengua Extranjera. Los sujetos procedían de las carreras de Ingeniería, Traducción, Periodismo, Biología, Química y Ciencias Políticas. Los sujetos del nivel A2 tenían como L1 alemán 16 (42\%), inglés 12 (32\%), francés 5, (13\%), portugués $2(5 \%)$, sueco $1(3 \%)$, italiano $1(3 \%)$ y holandés $1(3 \%)$. En el nivel B1, las L1 de los sujetos correspondían al inglés 12 (31\%), francés $11(28 \%)$, alemán $10(25 \%)$, portugués $3(7 \%)$, checo $1(3 \%)$, italiano $1(3 \%)$, sueco $1(3 \%)$.

Para el experimento, los sujetos se distribuyeron aleatoriamente en 2 grupos experimentales y uno control por cada nivel de competencia, como se ilustra en la Figura 1.

\section{Número sujetos}

Grupo/ Nivel

Experimental 1: FCE Indirecto

Experimental 2: FCE Directo

Control: Sin estrategias de FCE

TOTAL
A2

13

13

12

38
B1

14

13

12

39

Figura 1. Distribución de los grupos experimentales y control

\subsection{Selección de los Errores Enfocados en Este Estudio}

Para delimitar los errores que serían tratados por las estrategias de FCE, se realizó un análisis de errores asistido por computador de los 77 textos escritos por los sujetos de la muestra en el contexto del pre-test. Esto consistió en el procesamiento de los datos a través del software Uam Corpus Tool versión 3.2.

Para la identificación, clasificación y anotación de los errores, se procedió con los criterios y taxonomía de errores del modelo metodológico desarrollado por Autora (2014). Los resultados arrojaron que los errores más frecuentes identificados en el pre-test coinciden con los resultados encontrados en el estudio de Autora $(2014,2017)$, esto es, la ortografía acentual con un 53\% (agudas y esdrújulas), el uso 
de las preposiciones con un 23\% (a, de, en, por y para), la concordancia sintáctica con un 20\% (género y número en el sintagma nominal y de atributo con los verbos ser y estar). Acorde con ello, se determinó focalizarnos en los errores de ortografía acentual, preposiciones y concordancia gramatical para el tratamiento con el FCE.

\subsection{Estrategias de FCE en estudio}

Como se señaló anteriormente, dos son los tipos de estrategias de feedback correctivo en estudio, a continuación se ejemplifica la operacionalización de cada una de ellas.

1. Estrategias de FCE indirecto metalingüístico con información gramatical pero sin provisión de la respuesta correcta.

En mi país no hay (1) un equvelencia a este microrrelato. Pero tenemos un dicho; Más vale que parete cuando estás aún ganando. Cuando ganas mucho, los ojos del mundo se enfocan en ti. Y mucha gente quiere ver un error tuyo más que las victorías tuyas. Nadie es perfecto, y cuando has hecho muchas cosas buenas, los errores son mucho más visables o (2) notado.

(1): El determinante "un" tiene que expresar el mismo género gramatical del sustantivo al cual se refiere. El sustantivo "equivalencia" tiene género femenino.

(2): El atributo "notado" debe coincidir en número con el sujeto de la oración "los errores". El sujeto de la oración "los errores" expresa número plural.

Figura 2. Ejemplo de estrategias de FCE indirecto metalingüístico (Sujeto 25, B1, tarea 2)

2. Estrategias de FCE directo metalingüístico con información gramatical y provisión de la respuesta correcta.

La pintura chilena tiene muchas formas distintas. Los dos tipos que podriamosos ver ayer a la piñacoteca son la pintura mural y la pintura más (1) classica. La mayor parte del tiempo, la pintura mural respresenta, (2) critica o solo habla de la sociedad, se presenta con (3) muchas colores y muchas representacíones. El punto de la pintura classica, es mas de representar (algunas veces, perfectamente) la vida quotidiana, con pinturas de hombres o de paisajes.

(1), (2): iFíjate! Las palabras "clasicas" y "critica" son ESDRÚJULAS. Tienen el acento en la ANTEPENÚLTIMA SÍLABA: CLÁsicas, CRÍtica. No olvides que debes tildarlas siempre.

(3): El sustantivo "colores" es masculino. El determinante "muchas" debe expresar el mismo género del sustantivo "colores".

Figura 3. Ejemplo de estrategias de FCE directo metalingüístico (Sujeto 11, A2, tarea 4) 


\subsection{Procedimientos}

Como se visualiza en la figura 4 , el proceso de intervención lingüística considera la aplicación de un pre-test, la escritura de cuatro tareas, la revisión de los textos, la entrega del FCE y la aplicación de los post-test (correspondiente a la escritura de textos nuevos). A continuación, se explica cada uno de estos procedimientos.

\subsubsection{Aplicación del Pre-test y Análisis de Las Formas Lingüísticas}

La aplicación del pre-test se realizó en la semana 1 durante el desarrollo de las clases del curso de ELE de nivel A2 y B1, y consistió en la escritura de un texto narrativo sobre un tema cultural relacionado con los lugares turísticos del país de origen del estudiante. Las instrucciones para la tarea de escritura especificaban: la duración total de la tarea (40 minutos), la extensión del texto (150 para nivel A2 y 200 palabras para B1, como mínimo), la escritura en el bloc de notas para cautelar que el texto no fuera corregido automáticamente por un corrector y la prohibición del uso de traductores, internet o celulares con acceso a internet.

\subsubsection{Proceso de Escritura de Las Tareas (semana 2 a la 5)}

Las temáticas planteadas para la escritura fueron seleccionadas acorde con los contenidos tratados en los cursos A2 y B1 de ELE. De esta manera, la tarea de escritura 1 trataba sobre el comentario de un microrrelato revisado en clases, la tarea 2 sobre los problemas que afectan a los jóvenes, la tarea 3 sobre los problemas medioambientales del país de origen del estudiante y la tarea 4 sobre la pintura chilena. Cada tarea de escritura se integró en el marco de la clase, en función de la práctica de las 4 habilidades lingüísticas. La tarea de escritura se consideró en la planificación de la clase como la segunda actividad, es decir, se comenzaba con una pre-tarea cultural para activar los conocimientos temáticos aprendidos en las clases anteriores y luego los estudiantes escribían el texto en los computadores. Para ello, disponían de un total de 40 minutos. Al finalizar el texto, lo subían a un espacio del aula virtual del Programa de Español como lengua Extranjera ELE-UDEC (FERREIRA, 2013). Se cauteló entregar instrucciones similares para la escritura de todos los textos, para así mantener el nivel de dificultad de la tarea escrita por los estudiantes. 


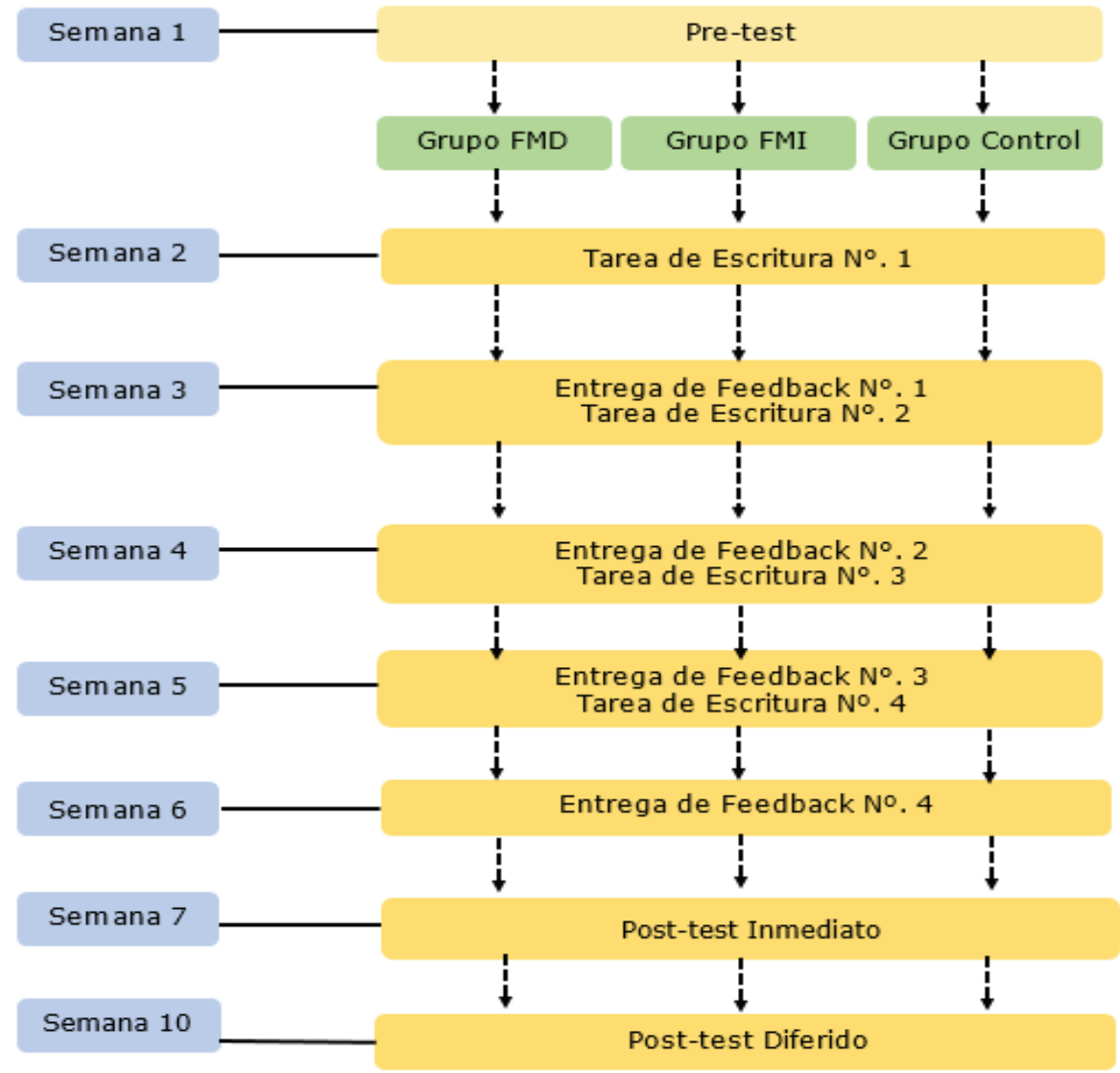

Figura 4. Modelo del proceso de intervención lingüística del FCE, Proyecto No. 1140651 (FERREIRA, 2014)

\subsubsection{Entrega del FCE durante Las Sesiones de Escritura}

Luego de que los estudiantes subían los textos al espacio virtual, dos ayudantes de investigación, tesistas del proyecto de investigación Fondecyt No. 1140651, revisaron los errores enfocados (ortografía acentual, preposiciones y concordancia gramatical) y proporcionaron las estrategias de FCE metalingüísticas indirectas y directas. Estas ayudantes fueron entrenadas en las estrategias de FCE y el tratamiento de errores. Cada error encontrado fue destacado con negrita, cursiva y se enumeró de manera consecutiva comenzando por el número 1, esto con el fin de agrupar por números los errores identificados en relación con la misma problemática. (Ver ejemplos en Figuras 2 y 3 ). 
Los estudiantes del grupo control sólo recibieron comentarios generales (culturales y léxicos) en relación con sus textos.

\subsubsection{Aplicación del Post-test Inmediato y Diferido}

Para la aplicación del post-test inmediato se consideró las mismas instrucciones del pre-test y se cauteló que el tema sobre el cual escribieran fuera similar. Así, en el pre-test escribieron sobre lugares turísticos del país de origen, en el post-test inmediato sobre lugares turísticos para visitar en Latinoamérica y en el post-test diferido sobre los lugares y actividades turísticas para realizar en Chile. El posttest diferido se realizó durante la semana 10, dos semanas después de concluido el proceso de intervención.

\section{ANÁLISIS Y DISCUSIÓN DE LOS RESULTADOS}

El experimento consideró una variable independiente y una dependiente. La variable independiente corresponde a cada uno de los tres grupos (2 experimentales y 1 control). La variable dependiente corresponde a la diferencia en la disminución de los errores enfocados en este estudio (ortografía acentual, las preposiciones y la concordancia gramatical) al comparar el pre-test y los post-test inmediato y diferido.

Para constatar si los grupos de los niveles A2 y B1 eran homogéneos antes del proceso de intervención, se aplicó el estadístico F Fisher. Los valores obtenidos al comparar los grupos en el nivel A2 (grupo experimental 1 y 2, $\mathrm{p}=0,05$; grupo experimental 1 y control, $\mathrm{p}=0,04$ grupo experimental 2 y control, $\mathrm{p}=0,4)$ y en el nivel B1 (grupo experimental 1 y 2, $\mathrm{p}=0,4$; grupo experimental 1 y control, $\mathrm{p}=0,3$; grupo experimental 2 y control, $\mathrm{p}=0,4$ ) indican que ambos grupos no presentan diferencias significativas y se consideran homogéneos.

\subsection{Hipótesis No.1 "Se evidenciará una mayor disminución de errores en los grupos experimentales con respecto al grupo control"}

Para corroborar la primera hipótesis se realizaron los contrastes entre los Grupos Experimentales y el Grupo Control en los niveles A2 y B1 de ELE.

3.1.1. Efectividad del FCE indirecto metalingüístico vs. el grupo control en el corto y largo plazo

En las Tablas 1 y 2 se presentan los resultados de los contrastes entre el grupo experimental 1 (con FCE indirecto metalingüístico) y el grupo control en los 
niveles A2 y B1 de ELE. Como se puede apreciar, en el post-test inmediato el grupo experimental 1 en promedio disminuyó en un mayor número sus errores (8.4 y 9.2) con respecto al grupo control (0.1 y 0.6) en los niveles A2 y B1, respectivamente.

Tabla 1. Diferencias en los contrastes del Grupo Experimental 1 para A2 y B1

\begin{tabular}{|c|c|c|c|c|c|c|c|c|c|c|c|c|c|c|c|c|c|c|c|c|c|c|c|c|c|c|c|c|c|}
\hline \multirow{2}{*}{ TEST } & \multicolumn{13}{|c|}{ Número de errores Nivel A2 } & \multirow{2}{*}{$\begin{array}{c}\text { Promedio } \\
\text { de } \\
\text { errores }\end{array}$} & \multicolumn{14}{|c|}{ Número de errores Nivel B1 } & \multirow{2}{*}{$\begin{array}{c}\text { Promedio } \\
\text { de } \\
\text { errores }\end{array}$} \\
\hline & s-1 & S-6 & S-9 & $\mathrm{s}-22$ & s-23 & $s-24$ & s-25 & S-31 & $S-32$ & $5-33$ & $S-40$ & S-41 & $s-42$ & & s-1 & S-2 & $s-12$ & S-13 & $\mid s-14$ & $s-15$ & $s-33$ s & $S-34 \mid S-$ & $S-40 \mid S$ & $s-41$ s & $s-45 \mid s$ & $s-46 \mid s$ & $\mid s-48 \mathrm{~s}$ & s-49 & \\
\hline \begin{tabular}{|c|} 
Diferencia Pre-test \\
Post-Inmediato
\end{tabular} & 10 & 14 & 19 & 13 & 13 & 5 & 9 & 2 & 12 & 3 & 0 & 3 & 6 & 8,4 & 16 & 5 & 11 & 7 & 13 & 5 & 6 & 8 & 11 & 9 & 19 & 7 & 6 & 6 & 9,2 \\
\hline \begin{tabular}{|c|} 
Diferencia Pre-test \\
Post-Diferido
\end{tabular} & 6 & 9 & 10 & 9 & 15 & 9 & 7 & 0 & 6 & 3 & 7 & 6 & 8 & 7,3 & 12 & 15 & 15 & 14 & 22 & 8 & 7 & 3 & 16 & 7 & 18 & 4 & 8 & 10 & 11,4 \\
\hline
\end{tabular}

Del mismo modo, en el post-test diferido se observa que el grupo experimental 1 logró en promedio una mayor disminución de los errores (7.3 y 11.4) en relación con el grupo control (0.2 y 2.6) en los niveles A2 y B1, respectivamente.

Tabla 2. Diferencias en los contrastes del Grupo Control, niveles A2 y B1

\begin{tabular}{|c|c|c|c|c|c|c|c|c|c|c|c|c|c|c|c|c|c|c|c|c|c|c|c|c|c|c|}
\hline \multirow{2}{*}{ TEST } & \multicolumn{12}{|c|}{ Número de errores Nivel A2 } & \multirow{2}{*}{\begin{tabular}{|c} 
Promedio \\
de \\
errores
\end{tabular}} & \multicolumn{12}{|c|}{ Número de errores Nivel B1 } & \multirow{2}{*}{$\begin{array}{c}\text { Promedio } \\
\text { de } \\
\text { errores }\end{array}$} \\
\hline & s-5 & $s-12$ & $s-13$ & $S-14$ & S-15 & $s-16$ & S-34 & $s-35$ & $s-36$ & $s-43$ & $s-44$ & s-45 & & s-7 & $s-20$ & $s-21$ & $s-22$ s & $s-23 \mathrm{~s}$ & $s-24$ s & $s-25$ & s-26 & $s-47$ & $s-50$ & S-51|s & S-52 & \\
\hline $\begin{array}{c}\text { Diferencia Pre-test } \\
\text { Post-Inmediato }\end{array}$ & 17 & -4 & -7 & 3 & -1 & 0 & -1 & 0 & 6 & 3 & 0 & -3 & 0,1 & 0 & -5 & -1 & -2 & 3 & 1 & 3 & 2 & -1 & 12 & -3 & -2 & 0,6 \\
\hline $\begin{array}{c}\text { Diferencia Pre-test } \\
\text { Post-Diferido }\end{array}$ & 22 & -5 & -5 & -2 & -11 & 4 & 1 & -4 & -3 & 3 & 1 & 1 & 0,2 & -2 & 5 & -4 & -4 & 2 & 1 & 3 & 2 & 2 & 16 & 7 & 3 & 2,6 \\
\hline
\end{tabular}

En el nivel A2, las diferemcias en los resultados del post-test inmediato entre el grupo experimental 1 y el grupo control presentan una validez estadística significativa $\left(\mathrm{S}_{\mathrm{c}}=35,1 ; \mathrm{t}\right.$-test $\left.=3,5 ; \mathrm{df}=23 ; \mathrm{p}<0,0009\right)$. Dichas diferencias entre estos dos grupos se mantienen estadísticamente significativas $\left(\mathrm{S}_{\mathrm{c}}=37,6\right.$; $\mathrm{t}$-test $=2,91 ; \mathrm{df}=23 ; \mathrm{p}<0,003)$ en el post-test diferido.

Así también en el nivel B1 las diferencias encontradas entre el grupo experimental 1 y el control tienen validez estadística, tanto en los resultados logrados en el post-test inmediato $(\mathrm{Sc}=18,6 ; \mathrm{t}$-test $=5,08 ; \mathrm{df}=24 ; \mathrm{p}<0,00001)$ como en el diferido $\left(\mathrm{Sc}_{\mathrm{c}}=29,8 ; \mathrm{t}\right.$-test $\left.=4,08 ; \mathrm{df}=24 ; \mathrm{p}<0,0002\right)$.

Estos resultados muestran de manera relevante que los estudiantes de los niveles A2 y B1 de ELE, que fueron apoyados con extrategias de FCE metalingüísticas indirectas, disminuyeron sus errores en la destreza escrita tanto en el corto como largo plazo con respecto al grupo control. La validez estadística significativa de dichos resultados evidencia en ambos niveles de competencia que estas estrategias contribuyen de manera notoria en el incremento y fortalecimiento de la precisión lingüística en la destreza escrita de los estudiantes de ELE. 
3.1.2. Efectividad del FCE directo metalingüístico vs. el grupo control en el corto y largo plazo

En lo que se refiere a los contrastes entre los resultados del grupo experimental 2 (con FCE directo metalingüístico) y los del grupo control en los niveles A2 y B1 de ELE, las Tablas 2 y 3 muestran las diferencias encontradas entre ambos grupos en el pre-test, post-test inmediato y post-test diferido.

Como se puede apreciar en la Tabla 3, el grupo experimental 2 en los niveles A2 y B1 disminuyó en promedio moderadamente el número de errores en el posttest inmediato (3.9 y 4.2) con respecto al grupo control (0.1 y 0.6$)$, como se evidencia en la Tabla 2. De igual forma, en el post-test diferido se observa que el grupo experimental 2 logró una disminución de los errores (2.8 y 4.4) en relación con el grupo control (0.2 y 2.6), respectivamente.

Tabla 3. Diferencias en los contrastes del Grupo Experimental 2 para A2 y B1

\begin{tabular}{|c|c|c|c|c|c|c|c|c|c|c|c|c|c|c|c|c|c|c|c|c|c|c|c|c|c|c|c|}
\hline \multirow{2}{*}{ TEST } & \multicolumn{13}{|c|}{ Número de errores Nivel A2 } & \multirow{2}{*}{$\begin{array}{c}\text { Promedio } \\
\text { de } \\
\text { errores }\end{array}$} & \multicolumn{12}{|c|}{ Número de errores Nivel B1 } & \multirow{2}{*}{$\begin{array}{c}\text { Promedio } \\
\text { de } \\
\text { errores }\end{array}$} \\
\hline & s-2 & S-3 & S-7 & $S-17$ & S-19 & $\mathrm{s}-20$ & S-21 & s-28 & $\mathrm{S}-29$ & $S-30$ & $\mathrm{~S}-38$ & \begin{tabular}{l|l}
$S-39$ & $S$
\end{tabular} & S-47 & & \begin{tabular}{|l|l} 
S-8 & S
\end{tabular} & \begin{tabular}{|l|l}
$S-10$ & $S$
\end{tabular} & \begin{tabular}{|l|l} 
S-16 & S
\end{tabular} & $s-17 \mid S-2$ & \begin{tabular}{l|l}
$S-27$ & $S-2$
\end{tabular} & \begin{tabular}{l|l|l}
-28 & $S-30$
\end{tabular} & \begin{tabular}{l|l}
-30 & $s-36$
\end{tabular} & $6 \mid s-37$ & $s-38$ & $3-39$ s & 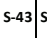 & s-44 & \\
\hline \begin{tabular}{|c|} 
Diferencia \\
Pre-test \\
\end{tabular} & -2 & -3 & 7 & -2 & 1 & 17 & 5 & 3 & 3 & 8 & 0 & 7 & 7 & 3,9 & 3 & 0 & 6 & 2 & 10 & 6 & 5 & 4 & 17 & -3 & -9 & 3 & 4,2 \\
\hline $\begin{array}{c}\text { Diferencia } \\
\text { Pre-test }\end{array}$ & 0 & -5 & 7 & 7 & 4 & 10 & -1 & -7 & 2 & 10 & -3 & 7 & 6 & 2,8 & 5 & 0 & 5 & 0 & 2 & 6 & 10 & 5 & 12 & 3 & -1 & 5 & 4,4 \\
\hline
\end{tabular}

Estas diferencias en la disminución de los errores fueron analizadas con la prueba t-Student para muestras independientes, registrándose los siguientes resultados:

- En el nivel A2, las diferencias observadas entre el grupo experimental 2 y el control solo presentan una validez estadística en el post-test inmediato $\left(\mathrm{S}_{\mathrm{c}}=\right.$; t-test $=; d f=; p<0,03)$. En el post-test diferido las diferencias no tuvieron significancia estadística.

- De manera similar, en el nivel B1 se obtuvo una validez estadística en el posttest inmediato $(\mathrm{Sc}=26,7 ; \mathrm{t}$-test $=1,76 ; \mathrm{df}=23 ; \mathrm{p}<0,04)$ para las diferencias encontradas entre el grupo Experimental 2 y el grupo Control; no así para el post-test diferido.

En consecuencia, en el corto plazo tanto en el nivel de competencia A2 como en el B1 se obtuvo una validez estadística moderada con respecto al grupo control en la corrección de errores a través de FCE directo metalingüístico. Esta situación puede explicarse por la naturaleza de este tipo de estrategia, por cuanto parece funcionar mejor cuando los estudiantes escriben un texto inmediatamente después 
de concluido el proceso de intervención lingüística, dado que ellos recuerdan mejor la corrección de sus errores transcurrido un tiempo breve después de la exposición a dichas estrategias. No obstante, dicho aprendizaje no se mantiene a lo largo del tiempo como se evidencia en los resultados del post-test diferido que no tuvieron validez estadística en ninguno de los dos niveles de competencia. Por otra parte, al parecer, los sujetos prestaron mayor atención a la entrega de la respuesta correcta que a la regla gramatical proporcionada en la clave metalingüística, por lo que no estarían internalizando ni transfiriendo dicho conocimiento lingüístico. Estos hallazgos son similares también con los de estudios previos (CHANDLER, 2003; BITCHENER \& KNOCH, 2010) en que se presenta un mejor comportamiento del FCE directo, inmediatamente concluido el proceso de intervención.

Según estos resultados, la hipótesis 1 se estaría cumpliendo en lo que respecta a la disminución de los errores en el grupo experimental 1 (apoyado con FCE indirecto metalingüístico) en relación con el grupo control (apoyado con comentarios generales sobre aspectos léxicos y culturales) tanto en el corto como largo plazo en ambos niveles de competencia (A2 y B1). Sin embargo, con respecto al grupo experimental 2 (apoyado con FCE directo metalingüístico) solo se estaría cumpliendo de manera parcial, por cuanto se obtuvo una validez estadística moderada en el corto plazo en ambos niveles de competencia (A2 y B1), no así mismo en el largo plazo donde las diferencias en la disminución de los errores con respecto al grupo control no tuvieron significancia estadística.

\subsection{Hipótesis No.2: Se evidenciará una mayor disminución en el proceso de corrección de los errores tratados con FCE indirecto metalingüístico que con el directo}

Para corroborar la segunda hipótesis se contrastó las diferencias observadas en cada test entre los grupos experimentales 1 y 2 con la finalidad de determinar si los estudiantes que fueron apoyados por FCE indirecto metalingüístico tendrían una mayor disminución en el proceso de corrección de sus errores que los tratados con FCE directo metalingüístico tanto en el corto como en el largo plazo.

\subsubsection{Efectividad del FCE indirecto metalingüístico vs. el directo en el corto plazo}

Las Tablas 1 y 3 dan cuenta de las diferencias entre el pre-test y el post-test inmediato en los grupos experimentales 1 y 2 en los niveles A2 y B1.

En la Tabla 1 se puede constatar que en el nivel A2, el grupo experimental 1, apoyado con estrategias indirectas metalingüísticas, disminuyó en un promedio 
de 8,4 errores en el post-test inmediato, mientras que el grupo experimental 2, que trabajó con estrategias directas metalingüísticas, disminuyó en un promedio de 3,9 errores (véase Tablas 1 y 3 ).

Del mismo modo, en el nivel B1 se puede observar que el grupo experimental 1 disminuyó en un promedio de 9,2 errores en el post-test inmediato, mientras que el grupo experimental 2 disminuyó en un promedio de 4,2 errores. Estos resultados muestran que en el corto plazo, tanto en los niveles A2 como en el B1, las estrategias de FCE indirectas metalingüísticas fueron más efectivas para tratar los errores de los aprendientes de ELE.

Para determinar si las diferencias encontradas eran estadísticamente significativas, se aplicó la prueba t-Student para muestras independientes. Los resultados muestran que las diferencias a favor de las estrategias metalingüísticas indirectas fueron significativas en ambos niveles:

- en el nivel A2 $\left(\mathrm{S}_{\mathrm{c}}=32,4 ; \mathrm{t}\right.$-test $\left.=1,77 ; \mathrm{df}=25 ; \mathrm{p}<0,04\right)$ y

- en el nivel B1 $\left(\mathrm{S}_{\mathrm{c}}=25,9 ;\right.$ t-test $\left.=2,5 ; \mathrm{df}=25 ; \mathrm{p}<0,008\right)$.

Por lo tanto, estos resultados reflejan que en el en el corto plazo habría una tendencia favorable en la corrección de errores a través de estrategias de FCE indirecto metalingüístico, tanto en el nivel principiante $\mathrm{A} 2(\mathrm{p}<0,04)$ como más notoriamente en el nivel intermedio B1 $(\mathrm{p}<0,008)$.

3.2.2. Efectividad del FCE indirecto metalingüístico vs. el directo en el largo plazo

De manera semejante, para observar el comportamiento de las estrategias metalingüísticas indirectas y directas en el largo plazo, se contrastó las diferencias obtenidas por los estudiantes entre el pre-test y el post-test diferido.

Según los resultados presentados en las Tablas 1 y 3, en el nivel A2 el grupo experimental 1 disminuyó en un promedio de 7,3 errores, mientras que el grupo experimental 2 sólo en un promedio de 2,8 errores. Del mismo modo, en el nivel B1 el grupo experimental 1 presenta una disminución en promedio de 11,4 errores y el grupo experimental 2 una diferencia en promedio de 4,4 errores.

Estos contrastes, en el aprendizaje de largo plazo muestran una tendencia a favor del uso de estrategias indirectas metalingüísticas por sobre las directas metalingüísticas tanto en el nivel A2 como en el B1:

- en el nivel A2 $\left(\mathrm{S}_{\mathrm{c}}=22 ; \mathrm{t}\right.$-test $\left.=2,3 ; \mathrm{df}=25 ; \mathrm{p}<0,01\right)$ con una validez estadística moderada y 
- en el nivel B1 $\left(\mathrm{S}_{\mathrm{c}}=22,7 ; \mathrm{t}\right.$-test $\left.=3,7 ; \mathrm{df}=25 ; \mathrm{p}<0,0004\right)$ con una validez estadística más robusta.

En resumen, los hallazgos aquí encontrados evidencian que se estaría cumpliendo la hipótesis 2, dado que las estrategias de FCE metalingüísticas indirectas fueron más efectivas que las directas para apoyar la disminución de los errores en la destreza escrita de los aprendientes de ELE en ambos niveles. Como lo señalábamos en la fundamentación teórica, el FCE indirecto estimula a los aprendientes de ELE a reflexionar sobre las formas lingüísticas (ELLIS, 2009) y los ayuda a desarrollar habilidades para llegar a ser "autoeditores independientes" (HYLAND \& HYLAND, 2006; SHEEN, 2011; VAN BEUNINGEN, 2010).

\section{CONCLUSIONES}

Como se señaló anteriormente en la introducción de este trabajo, un número importante de aprendientes de ELE presenta importantes debilidades en sus niveles de precisión gramatical en la destreza escrita, incluso cuando muestran altos rendimientos de fluidez lingüística. Este artículo ha centrado su atención en responder la pregunta sobre si las estrategias de FCE indirecto metalingüístico y directo metalingüístico son efectivas para apoyar la disminución de errores de ortografía acentual, concordancia gramatical y preposiciones en la destreza escrita del Español como lengua Extranjera.

En general, se pudo corroborar la hipótesis 1 en lo referido a los resultados de las estrategias utilizadas para apoyar los errores en los grupos experimentales que trabajaron con feedback indirecto metalingüístico en los niveles A2 y B1 tanto en el corto como en el largo plazo. No obstante, pudimos corroborarla solo parcialmente en ambos grupos y niveles (A2 y B1) que trabajaron con feedback correctivo escrito directo metalingüístico en el corto plazo. En lo que respecta a la hipótesis 2 de este estudio fue corroborada cabalmente en ambos niveles de competencia tanto en el corto como en el largo plazo. Si bien se observó una mayor robustez estadística en los resultados del nivel B1.

Los resultados de este estudio muestran una mayor efectividad del FCE indirecto metalingüístico con respecto al directo en ambos niveles y con respecto a trabajar sin apoyo de FCE (control) en el caso del nivel B1. Esto significa que, en general, el feedback correctivo escrito tuvo un mejor desempeño en el nivel de competencia intermedio (B1) que en el principiante (A2). Es decir, los resultados en el nivel B1 son significativamente más robustos que en el nivel A2. En efecto, 
aun cuando se percibe una tendencia interesante en el uso de estas estrategias en el nivel A2, dado que muestran un mayor efecto en el mejoramiento de los errores que el FCE directo, se necesita mayor evidencia para corroborar estadísticamente esta tendencia. La problemática podría explicarse a nivel de la complejidad del conocimiento metalingüístico encubierto en la estrategia y/o en la capacidad de retención de dicho conocimiento gramatical por parte de los sujetos para procesar la información desplegada en la clave metalingüística.

En lo que respecta al nivel B1, los resultados muestran una sólida validez estadística, evidenciando una notoria efectividad de las estrategias de FCE indirectas metalingüísticas tanto en el corto como largo plazo. Estos resultados son consecuentes con los evidenciados en la literatura del FCE (FERRIS, 2010; SHEEN, 2011). Por cuanto los sujetos de mayor competencia (B1) pueden procesar y retener mejor la información metalingüística entregada en las estrategias indirectas, dado que cuentan con mayores recursos lingüísticos para llevar a cabo dicho procesamiento y con ello lograr una mayor retención de lo aprendido. En el proceso de intervención, los alumnos de nivel B1 de ELE recibieron la información gramatical que les permitió procesar los errores por sí mismos y ser más asertivos en las siguientes situaciones en que se requería usar dichos recursos en las tareas de escritura. Por consiguiente, el uso de estrategias de FCE indirecto promueve un rol más activo en un estudiante competente a nivel lingüístico, puesto que está en condiciones cognitivas para procesar la información lingüística pertinente entregada para auto-reparar su error (FERREIRA, 2003).

Los resultados aquí logrados permiten visualizar que ante las limitaciones de un input comprensible para ofrecer evidencia negativa acerca de los enunciados no permisibles en la lengua, el tratamiento de los errores por medio de estrategias de feedback correctivo escrito indirecto metalingüístico y directo metalingüístico facilita el proceso de adquisición y mejoramiento de los errores en la escritura. Ahora bien, es necesario realizar nuevos estudios que repliquen esta experiencia para confirmar las tendencias que se han obtenido en esta investigación.

En el ámbito de español como lengua extranjera y/o segunda lengua, los hallazgos encontrados en la presente investigación son una contribución interesante, por cuanto no solo han demostrado la efectividad del FCE metalingüístico indirecto sobre la corrección de los errores, sino también han proporcionado las bases para futuras investigaciones cuyos focos sean la efectividad del FCE sobre el aprendizaje o usos correctos en la destreza escrita. 


\section{REFERENCIAS BIBLIOGRÁFICAS}

BITCHENER, J. (2009). Measuring the effectiveness of written corrective feedback: A response to "Overgeneralization from a narrow focus: A response to Bitchener (2008)". Journal of Second Language Writing, 18, pp. 276-279.

BITCHENER, J. (2012). A reflection on the 'language learning potential' of written CF. Journal of Second Language Writing, 21, pp. 348-363.

BITCHENER, J \& FERRIS, D. (2012). Written Corrective Feedback in Second Language Acquisition and Writing. New York: Routledge.

BITCHENER, J., \& KNOCH, U. (2009a). The value of a focused approach to written corrective feedback. ELT Journal, 63, pp. 204-211.

BITCHENER, J., \& KNOCH, U. (2009b). The relative effectiveness of different types of direct written corrective feedback. System, 37, pp. 322-329.

BITCHENER, J. \& KNOCH, U. (2010). The contribution of written corrective feedback to the language development: A ten-month study. Applied Linguistics 31, No2, (2010): pp. 193-214.

CHANDLER, J. (2003). The efficacy of various kinds of error feedback for improvement in the accuracy and fluency of L2 student writing. Journal of second language writing, 12(3), pp. 267-296.

CHAUDRON, C. (1977). A descriptive model of discourse in the corrective treatment of learners' errors. Language Learning, 27, pp. 29-46.

ELLIS, R. (2016). Focus on form: A critical review. Language Teaching Research, Vol. 20, 3, pp. pp. $405-428$.

ELLIS, R (2009). Cognitive feedback and teacher development. L2 Journal, 1, pp. 3-18.

ELLIS, R. (2008). A typology of written corrective feedback types. ELT Journal. 63, pp. 97-107.

ELLIS, R., SHEEN, Y., MURAKAMI, M., \& TAKASHIMA, H. (2008). The effects of focused and unfocused written corrective feedback in an English as a foreign language context. System, 36(3), pp. 353-371. 
FERREIRA, A. (2014). El feedback correctivo escrito directo e indirecto en la adquisición y aprendizaje del ELE. Proyecto de Investigación Fondecyt No. 1140651.

FERREIRA, A. (2014). Análisis de Errores Asistido por Computador basado en un Corpus de Aprendientes de Español como Lengua Extranjera. Revista Signos, 47(86), pp. 385-411.

FERREIRA, A., VINE, A. \& ELEJALDE, J. (2013). Hacia una prueba de nivel en español como lengua extranjera. Revista de Linguística Teórica y Aplicada, 51(2), pp. 73-103.

FERREIRA, A (2007). Estrategias efectivas de feedback correctivo para el aprendizaje de lenguas asistido por computadores. Revista Signos, 40(65), pp. 521-544.

FERREIRA, A., MOORE, D.J., \& MELLISH, C. (2007). A study of feedback strategies in foreign language classrooms and tutorials with implications for Intelligent Computer Assisted language learning systems. International Journal of Artificial Intelligence in Education, 17(4), pp. 389-422.

FERREIRA, A. (2006). Estrategias de feedback positivo y correctivo en el español como lengua extranjera. Revista Signos, 39(62), pp. 379-406.

FERREIRA, A. (2003). Feedback strategies for second language teaching with implications for Intelligent Tutorial Systems, Ph.D. Thesis, University of Edinburgh, UK.

FERRIS, D. (2004). The "grammar correction" debate in L2 writing: Where are we, and where do we go from here? (and what do we do in the meantime...? ). Journal of second language writing, 13(1), pp. 49-62.

FERRIS, D. (2010). Second language writing research and written corrective feedback in SLA Intersections and Practical Applications. Studies in Second Language Acquisition, 32, pp. 181-201.

FERRIS, D. (2012). Written Corrective Feedback in Second Language Acquisition and Writing studies. Language Teaching, 45(4), pp. 446-459.

FERRIS, D. \& ROBERTS, B. (2001). Error feedback in L2 writing classes: How explicit does it need to be? Journal of second language writing, 10(3), pp. 161-184.

HYLAND, K. \& HYLAND, F. (2006). Feedback on second language students' writing. Language Teaching, 39(2), pp. 83-101. 
LONG M. \& ROBINSON P. (1998). Focus-on-form: Theory, research and practice. In: Doughty C., Williams J.(Eds.), Focus-on-form in classroom second language acquisition (pp. 15-41). Cambridge: Cambridge University Press.

LOEWEN, S. (2012). The role of feedback. In S. Gass \& A. Mackey (Eds.), The Routledge Handbook of Second Language Acquisition (pp.24 -40). Abingdon: Routledge.

SHEEN, Y. (2007). The effect of focused written corrective feedback and language aptitude on ESL learners' acquisition of articles. Tesol Quarterly, 41(2), pp. 255-283.

SHEEN, Y. (2011). Corrective Feedback Individual Differences and Second Language Learning. New York: Springer.

SCHMIDT, R. (2010). Attention, awareness, and individual differences in language learning. In W. M. Chan, S. Chi, K. N. Cin, J. Istanto, M. Nagami, J. W. Sew, T. Suthiwan, \& I. Walker, Proceedings of CLaSIC 2010, Singapore, December 2-4 (pp. 721 737). Singapore: National University of Singapore, Centre for Language Studies.

VAN BEUNINGEN, C. (2010). Corrective feedback in L2 writing: Theoretical perspectives, empirical insights, and future directions. International Journal of English Studies, 10(2), pp. 1-27.

Recebido: $14 / 08 / 2017$

Aceito: 02/11/2017 